\title{
Solubility of amphiphiles in membranes: influence of phase properties and amphiphile head group
}

\author{
Luís M.B.B. Estronca, ${ }^{\mathrm{a}}$ Maria João Moreno, ${ }^{\mathrm{a}}$ Magda S.C. Abreu, ${ }^{\mathrm{a}}$ \\ Eurico Melo, ${ }^{b, c}$ and Winchil L.C. Vaz ${ }^{a, *}$ \\ a Departamento de Química, Universidade de Coimbra, Coimbra 3004-535, Portugal \\ b Institute de Tecnologia Química e Biológica, Universidade Nova de Lisboa, Oeiras 2784-505, Portugal \\ c Institute Superior Técnico, Lisboa 1049-001, Portugal
}

Received 17 July 2002

\begin{abstract}
The solubilities of two fluorescent lipid amphiphiles with comparable apolar structures and different polar head groups, NBDhexadecylamine and RG-tetradecylamine (or -octadecylamine), were compared in lipid bilayers at a molar ratio of $1 / 50$ at $23^{\circ} \mathrm{C}$. Bilayers examined were in the solid, liquid-disordered, or liquid-ordered phases. While NBD-hexadecylamine was soluble in all the examined bilayer membrane phases, RG-tetradecylamine was stably soluble only in the liquid-disordered phase. RG-tetradecylamine insolubility in solid and liquid-ordered phases manifests itself as an aggregation of the amphiphile over a period of several days and the kinetics of aggregation were studied. Solubility of these amphiphiles in the different phases examined seems to be related to the dipole moment of the amphiphile (in particular, of the polar fluorophore) and its orientation relative to the dipolar potential of the membrane. We propose that amphiphilic molecules inserted into membranes (including lipid-attached proteins) partition into different coexisting membrane phases based upon: (1) nature of the apolar structure (chain length, degree of saturation, and chain branching as has been proposed in the literature); (2) magnitude and orientation of the dipole moment of the polar portion of the molecules relative to the membrane dipolar potential; and (3) hydration forces that are a consequence of ordering of water dipoles at the membrane surface. (c) 2002 Elsevier Science (USA). All rights reserved.
\end{abstract}

Keywords: Fluorescent lipid amphiphiles; Amphiphile aggregation; Membrane phases; Phase partitioning; Dipolar surface potential

The increasing acceptance that biological membranes are heterogeneous chemical systems [1-7] whose heterogeneity has physiological consequences [3,6-9] makes the detection and quantitation of lateral heterogeneity in biological membranes a matter of paramount interest. Fluorescence microscopy is one of the best available techniques to study membrane heterogeneity, since heterogeneous distribution of proteins may be visualized by the use of fluorescent constructs of specific membrane proteins [10] while lipid heterogeneity may be visualized

\footnotetext{
is Abbreviations: DPPC, 1,2-dipalmitoylphosphatidylcholine; FLA, fluorescent lipid amphiphile(s); MLV, multilamellar vesicle(s); NBD, $N$-(7-nitrobenzoxa-2,3-diazol-4-yl); POPC, 1-palmitoyl-2-oleoylphosphatidylcholine; RG, a carboxyrhodamine-110 derivative marketed by Molecular Probes, as Rhodamine Green.

${ }^{*}$ Corresponding author. Fax: +351-239827703.

E-mail address: wvaz@ci.uc.pt (W.L.C. Vaz).
}

by the use of fluorescent lipid amphiphiles (FLA). In particular, the increasing perfection of high-resolution optical microscopic techniques [11-15] can be expected to make these approaches powerful tools for the cell biologist in the near future.

FLA, by the nature of their physical properties, partition into membranes from an aqueous phase but their partitioning between coexisting membrane phases (domains) is governed by the physical order and chemical properties of the lipid domains as well as the physical and chemical properties of the FLA. The rational use of FLA as markers of membrane lipid heterogeneity requires a quantitative understanding of how a given FLA will partition in a membrane with more than one coexisting phase. We [16-19] have therefore attempted to obtain quantitative information, in terms of partition coefficients and the thermodynamics and kinetics of association of FLA with membrane systems 
in which there is phase coexistence (heterogeneity). Several other approaches have also been used to address this problem (for a recent review see [20]). The consensus at the present time is that FLA with long saturated acyl/ alkyl chains preferentially partition into more ordered membrane domains while chain unsaturation or short chains lead to preferential partitioning into less ordered domains. An aspect that has been ignored in previous work is the effect of the nature of the membrane-water interface and the chemical properties of the polar portion of the FLA upon its solubility in a given type of membrane domain and its partitioning between different membrane phases.

In the present paper, we address the question of how the polar portion of an FLA influences its solubility in a lipid bilayer membrane. In a previous report [16], we had shown that the partitioning of NBD-alkylamines between coexisting solid and liquid-disordered phases was determined primarily by the length of the alkyl chain and its degree of saturation. Short, fully saturated chains $\left(\mathrm{C}_{12: 0}\right)$ and long chains with a c/s-unsaturation $\left(\mathrm{C}_{18: 1-(c i s)}\right)$ partitioned preferentially into disordered phase domains whereas long fully saturated chains showed no phase preference. Previous work by other authors (for a review see [20]) had shown that in some cases long saturated chain FLA $\left(\mathrm{C}_{16: 0}\right.$ through $\left.\mathrm{C}_{24: 0}\right)$ actually preferred ordered phase domains. We have now compared the solubility of NBD-alkylamines (NBD-C $\mathrm{C}_{16: 0}$ ) and rhodamine green-alkylamines ( $\mathrm{RG}-\mathrm{C}_{14: 0}$ and $\left.\mathrm{RG}-\mathrm{C}_{18: 0}\right)$ in lipid bilayers prepared from pure DPPC (solid phase) and pure POPC (liquid-disordered phase), and from a mixture of both these phosphatidylcholines with cholesterol at 30 $\mathrm{mol}^{\%}$ (liquid-ordered phases). In these FLA, the fluorophores constitute the polar portion of the amphiphiles and their apolar portions are comparable so that the relative solubilities can be attributed entirely to the nature of the polar portion and its interaction with its local membrane environment. It is proposed that one of the factors that could condition probe solubility in a given phase is the dipolar potential of the membrane surface and the orientation and/or magnitude of the dipole moment of the polar portion of the FLA. The possible consequences of these interactions for the localization of lipid-anchored proteins in coexisting membrane domains, such as in "rafts" [3], are discussed.

\section{Materials and methods}

The phospholipids DPPC, POPC, and 1-palmitoyllysophosphatidylcholine were from Avanti Polar Lipids, Alabaster, USA. Cholesterol was from Serva Fine Biochemicals, Heidelberg, DE. NBD-C $\mathrm{C}_{16: 0}$ and rhodamine green carboxylic acid $N$-hydroxysuccinimidyl ester hydrochloride "mixed isomers" were from Molecular Probes, Eugene, USA. Tetradecylamine and octadecylamine were obtained from Sigma-Aldrich Chemie, Madrid, ESP. All reagents were of the highest commercially available purity. Solvents of analytical reagent grade were from Merck Portuguesa, Lisbon, PT.
RG- $\mathrm{C}_{14: 0}$ and $\mathrm{RG}-\mathrm{C}_{18: 0}$ were synthesized by the addition of a chloroform/methanol $(1 / 1, \mathrm{v} / \mathrm{v})$ solution of the desired alkylamine (10-fold molar excess over dye reagent) to a dimethylformamide solution of the dye reagent (rhodamine green carboxylic acid $N$-hydroxysuccinimidyl ester hydrochloride "mixed isomers"), which contained a few grains of anhydrous sodium carbonate. The reaction mixture was vortexed and allowed to stand for $24 \mathrm{~h}$ at room temperature after which the desired product (RG-alkylamide) was isolated and purified by preparative thin layer chromatography on Silica Gel 60 plates (Merck Portuguesa, Lisbon, PT) using chloroform/methanol (1/1, v/v) as eluant.

Phospholipid concentration in aqueous suspensions was determined by the method of Bartlett [21] and cholesterol was determined by the Liebermann-Burchard method [22]. FLA concentrations in methanol solution were determined by spectrophotometry using a molar extinction coefficient of $77,500 \mathrm{M}^{-1} \mathrm{~cm}^{-1}$ for $\mathrm{RG}$ at $502 \mathrm{~nm}$ and of $24,000 \mathrm{M}^{-1} \mathrm{~cm}^{-1}$ for NBD at $466 \mathrm{~nm}$.

In the preparation of MLV, a solution of the desired lipid or lipid/ FLA mixture in chloroform/methanol $(1 / 1, v / v)$ was evaporated by blowing dry nitrogen over the heated (by blowing hot air onto the external surface of the tube) solution. The residue was then left in a vacuum dessicator for at least $8 \mathrm{~h}$ at $23^{\circ} \mathrm{C}$. The solvent-free residue, heated in a water bath at $60^{\circ} \mathrm{C}$, was hydrated at this temperature with $10 \mathrm{mM}$ MOPS buffer, $\mathrm{pH} 7.5$, which had been previously heated to the same temperature. The tube was maintained at $60^{\circ} \mathrm{C}$ for $5 \mathrm{~min}$ and then vigorously vortexed for $1 \mathrm{~min}$ after which it was cooled to $23^{\circ} \mathrm{C}$ by immersion in a water bath at this temperature.

Absorption spectra of the FLA in liposome suspensions were obtained at room temperature $\left(23^{\circ} \mathrm{C}\right)$ on a Unicam UV530 spectrophotometer using $1 \mathrm{~cm}$ path-length quartz cuvettes. The dispersion of the MLV suspensions $\left(1.1 \times 10^{-4} \mathrm{M}\right.$ in total lipid $)$ and the relatively low absorbance of the FLA inserted into them required a subtraction of the contribution of dispersion to the experimentally obtained absorption spectra. To do this, the Microcal Origin program was used to generate a line described by a polynomial expression of the third degree that fitted best to the values of absorbance between 400 and $420 \mathrm{~nm}$ and between 650 and $700 \mathrm{~nm}$ (for samples containing the $\mathrm{RG}$ chromophore). It was previously verified that the dispersion spectra of MLV dispersions without any chromophore in them could be very well described by this procedure. Since the RG chromophore does not absorb significantly between 650 and $700 \mathrm{~nm}$ and absorbs only very little between 400 and $420 \mathrm{~nm}$, this line serves as a dispersion baseline for the experimentally obtained absorption spectrum. The dispersion-free absorption spectra are then obtained by subtraction of the experimental absorption spectra from the simulated dispersion spectra.

Fig. 1 shows the chemical structures of the FLA used in this work. Their minimum energy conformations and theoretical dipole moments

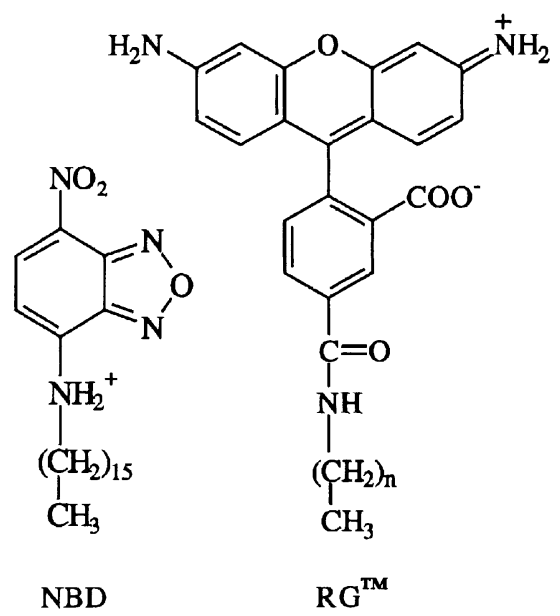

Fig. 1. Chemical structures of the fluorescent lipid amphiphiles used in this work. 
were calculated using the HyperchemVersion 5 package and the AMI semi-empirical method. The molecules were considered to be in a vacuum.

\section{Results and discussion}

Absorption and emission spectral characteristics of NBD$C_{16: 0}$ and $R G-C_{14: 0}$ in aqueous solution and in micellar solutions of lysophosphatidylcholine

The fluorescent reagent, rhodamine green (RG) $N$-hydroxysuccinimidyl ester, obtained from Molecular Probes, and NBD-ethanolamine, prepared by reaction of ethanolamine with NBD-chloride, were used as water-soluble references for the absorption and emission spectra. Their spectra in aqueous solution buffered at pH 7.5 are shown in Fig. 2A. The absorption and emission spectra were obtained in methanol solutions as well (data not shown). For RG, the absorption maximum is blue-shifted by about $4-6 \mathrm{~nm}$ and the emission spectral maximum is the same in water compared to methanol. For NBD-ethanolamine, the absorption and emission spectral maxima are red-shifted by 11 and $14 \mathrm{~nm}$, respectively, in water compared with methanol. The absorption and emission spectra of NBD-C $\mathrm{C}_{16: 0}$ and $\mathrm{RG}-\mathrm{C}_{14: 0}$ (both with alkyl chains) inserted into micelles of palmitoyllysophosphatidylcholine at a probe to micelle ratio of 0.1 (assuming an aggregation number of 100 for the micelles) are shown in Fig. 2B. The spectral shapes are comparable to those seen for aqueous solutions of the water-soluble dyes but the maxima are redshifted in the absorption as well as emission spectra for RG-C $\mathrm{C}_{14: 0}$, whereas NBD- $\mathrm{C}_{16: 0}$ shows no shift of the absorption spectral maximum but a blue-shift of $16 \mathrm{~nm}$ in the emission maximum. Some relevant spectral information is presented in Table 1.

\section{Absorption and emission spectral characteristics of $N B D$ -} $C_{16: 0}$ and $R G-C_{14: 0}$ inserted into lipid bilayer membranes

Fig. 3 shows the absorption spectra of RG-C $14: 0$ inserted into MLV of POPC (liquid-disordered phase), DPPC (solid or gel phase), POPC/cholesterol (7/3 molar
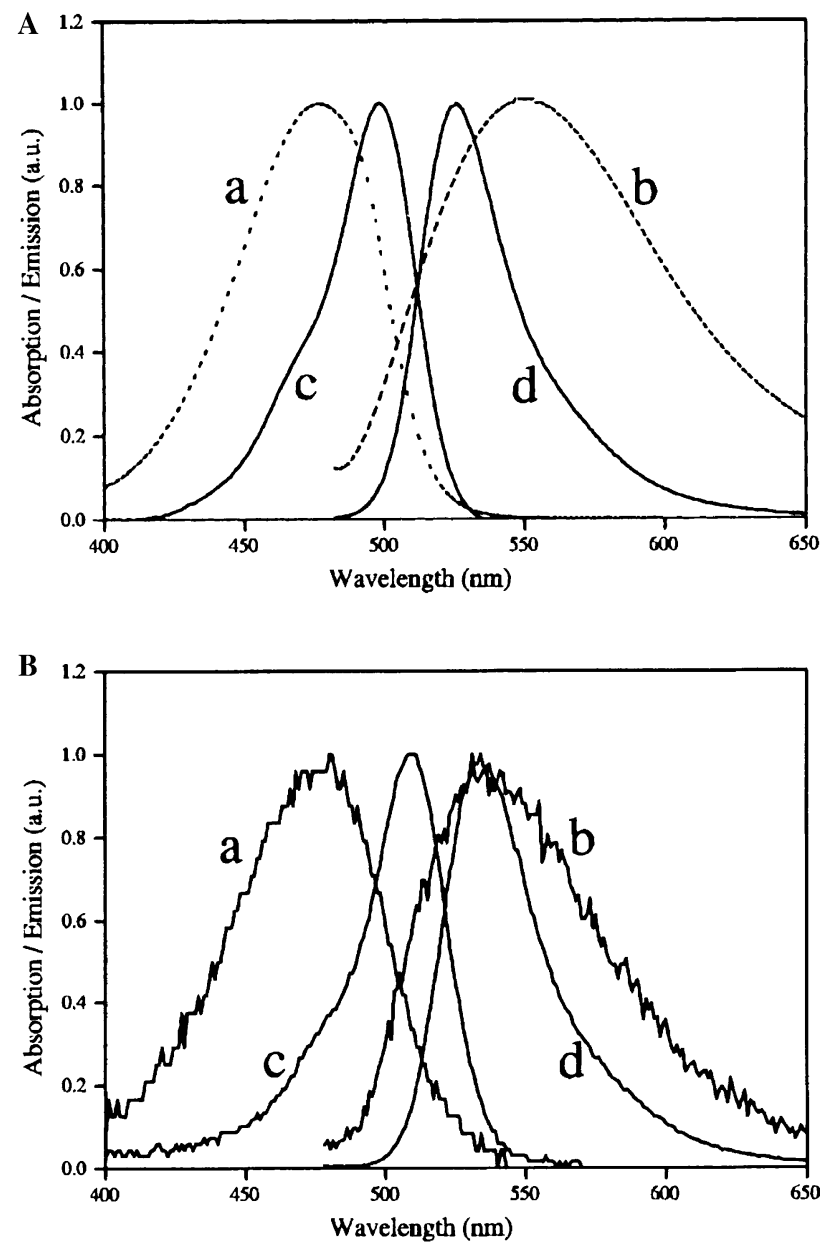

Fig. 2. Absorption and fluorescence emission spectra of (A) NBDethanolamine ( $\mathrm{a}$ and $\mathrm{b}$ ), and RG- $N$-hydroxysuccinimidyl ester (c and d); both in $10 \mathrm{mM}$ MOPS buffer, $\mathrm{pH}$ 7.5. (B) NBD-hexadecylamine (a and b), and RG-tetradecylamine (c and d) in 1-palmitoyllysophosphatidylcholine micelles at a molar ratio of $10^{-3}$ in $10 \mathrm{mM}$ MOPS buffer, $\mathrm{pH} 7.5$.

ratio), and DPPC/cholesterol (7/3 molar ratio), all at an FLA/host lipid ratio of $1 / 50$, and at a temperature of $23^{\circ} \mathrm{C}$. Spectra were obtained, as described in the Materials and methods over a period of time extending over up to $120 \mathrm{~h}$.

In MLV made from pure POPC (Fig. 3A), the absorption spectrum of $\mathrm{RG}-\mathrm{C}_{14: 0}$ is similar to that seen for

Table 1

Some spectroscopic characteristics of the probes used in this work

\begin{tabular}{|c|c|c|c|c|c|c|c|c|c|}
\hline \multirow[t]{2}{*}{ Fluorescent probe } & \multicolumn{3}{|c|}{$\lambda_{\text {absorption }}($ maximum), $\mathrm{nm}$} & \multicolumn{3}{|c|}{$\lambda_{\text {emission }}($ maximum $), \mathrm{nm}$} & \multicolumn{3}{|c|}{ Relative quantum yield ${ }^{\mathrm{a}}$} \\
\hline & Water & Methanol & Lyso-PC & Water & Methanol & Lyso-PC & Water & Methanol & Lyso-PC \\
\hline RG-reagent & 498 & 502 & - & 526 & 526 & - & 0.21 & 0.91 & - \\
\hline NBD-ethanolamine & 476 & 465 & - & 551 & 537 & - & 0.04 & 0.36 & - \\
\hline NBD-C $16: 0$ & 466 & 467 & 476 & - & 532 & 535 & - & 0.37 & 0.27 \\
\hline
\end{tabular}

${ }^{\mathrm{a}}$ Note. The relative quantum yields are given with reference to the quantum yield of $\mathrm{RG}-\mathrm{C}_{14}$ in methanol. No fluorescence emission was detected when RG-C $\mathrm{C}_{14: 0}$ was excited at $608 \mathrm{~nm}$.

${ }^{\mathrm{b}}$ Excitation at $516 \mathrm{~nm}$. 

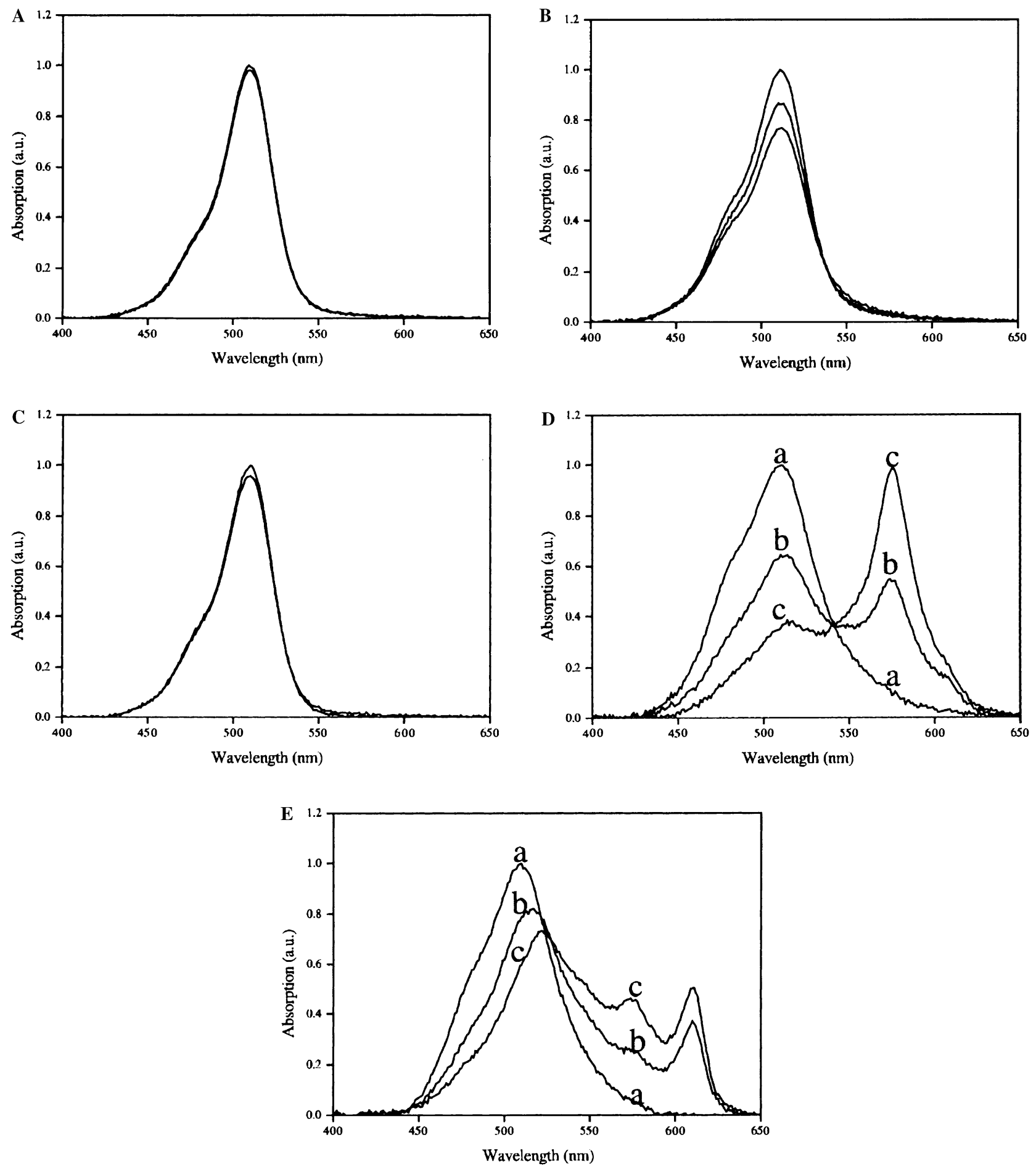

Fig. 3. Absorption spectra, at $23{ }^{\circ} \mathrm{C}$, of RG-tetradecylamine inserted into MLV prepared from (A) pure POPC at $t=10$ min (upper trace) and $t=50 \mathrm{~h}$; (B) pure DPPC at $t=10 \mathrm{~min}$ (upper trace), $t=6.5 \mathrm{~h}$ (middle trace), and $t=48 \mathrm{~h}$ (lowest trace); (C) POPC/cholesterol (7/3, molar ratio) at $t=10 \mathrm{~min}$ (upper trace) and $t=47 \mathrm{~h}$; (D) DPPC/cholesterol (7/3, molar ratio) at $t=10 \mathrm{~min}$ (upper trace, a), at $t=6.7 \mathrm{~h}$ (middle trace, b), and $t=96 \mathrm{~h}$ (lowest trace, c); and (E) of RG-octadecylamine inserted into MLV prepared from DPPC/cholesterol (7/3, molar ratio) at $t=10 \mathrm{~min}$ (upper trace, a), at $t=9.2 \mathrm{~h}$ (middle trace, b), and at $t=96 \mathrm{~h}$ (lowest trace, c). The molar ratio of the fluorescent lipid amphiphile to the host lipid was $1 / 50$ in all cases.

this FLA inserted into micelles of lysophosphatidylcholines at a concentration of less than 0.1 FLA per micelle (see Fig. 2B). The spectrum remains unchanged after over $95 \mathrm{~h}$ at $23^{\circ} \mathrm{C}$. Excitation of the fluorophore at any wavelength within this absorption band results in a fluorescence emission spectrum whose spectral distribution is characteristic for the emission from dilute solutions of RG- $\mathrm{C}_{14: 0}$ inserted into lysophosphatidylcholine 
micelles. We conclude that the FLA is monomerically dispersed in these lipid bilayers and remains stable in that state over the time examined. In MLV prepared from DPPC (Fig. 3B), the absorption spectrum of RG-C $\mathrm{C}_{14: 0}$ is qualitatively similar to monomerically dispersed FLA spectra seen in micelles of lysophosphatidylcholine and MLVs of POPC soon after the preparation of the MLV dispersions but over time there is a decrease in the intensity of the absorption band with maximum at $510 \mathrm{~nm}$ and a concomitant increase in absorbance between 540 and $600 \mathrm{~nm}$, a clear definition of the absorption maximum not being possible from the data.

In MLV prepared from a mixture of POPC and cholesterol (7/3 molar ratio) (Fig. 3C), the absorption spectrum, shortly after the preparation of the sample, is identical to the spectrum in MLV prepared from pure POPC. However, there is a slight decrease in the absorption band with maximum at $510 \mathrm{~nm}$ over time that is accompanied by the appearance and time-dependent increase in the intensity of an absorption band in the $540-600 \mathrm{~nm}$ region. The same behavior, but considerably more accentuated, is seen in MLV prepared from a mixture of DPPC and cholesterol (7/3 molar ratio) (Fig. $3 \mathrm{D})$. It is of interest to note that the excitation of the fluorophore in the absorption band with a maximum at $575 \mathrm{~nm}$ does not result in a detectable fluorescence emission.

We interpret the time-dependent appearance of the absorption band with maximum at $575 \mathrm{~nm}$ in the bilayers containing $\mathrm{RG}-\mathrm{C}_{14: 0}$ to be due to some aggregated state of the FLA in the lipid bilayer. Similar absorption bands are seen for aqueous solutions of $\mathrm{RG}-\mathrm{C}_{14: 0}$ (data not shown).

When $\mathrm{RG}-\mathrm{C}_{18: 0}$ is dissolved at a molar ratio (relative to the host lipid) of $1 / 50$ in MLV prepared from a mixture of DPPC and cholesterol (7/3 molar ratio), the time-dependent reduction in intensity of the absorption band with a maximum at $510 \mathrm{~nm}$ is accompanied by the appearance and time-dependent increase of two new absorption bands with maxima at 575 and $610 \mathrm{~nm}$ (Fig. $3 \mathrm{E})$. The band with a maximum at $610 \mathrm{~nm}$ becomes less intense when the ratio of FLA to host lipid is $1 / 62.5$ and does not appear at all when the ratio is $1 / 250$. The timedependent appearance of the absorption bands at 575 and $610 \mathrm{~nm}$ are interpreted to be due to the formation of different aggregation states of the FLA in the lipid bilayers, the form absorbing with a maximum at $610 \mathrm{~nm}$ not being possible at low concentrations of the FLA in the bilayer. It is interesting to note that the band at $610 \mathrm{~nm}$ does not appear in the case of $\mathrm{RG}-\mathrm{C}_{14: 0}$ under identical conditions, implying that this aggregation state is somehow dependent upon the chain length of the FLA. Excitation in the absorption bands in the 575 and $610 \mathrm{~nm}$ region does not result in fluorescence emission.

When NBD- $\mathrm{C}_{16: 0}$ is inserted into any of the lipid bilayers discussed above, under the same conditions, no time-dependent changes in the absorption or in the fluorescence emission spectra are detected (data not shown), implying that the monomerically dispersed NBD-FLA is stably soluble and shows no tendency to aggregate in all the bilayers and at the concentrations ( $2 \mathrm{~mol} \%$ FLA or lower) examined.

Time-dependence of the $R G$ - $C_{X: 0}$ aggregation process in lipid bilayers

We have followed the time-dependence of the aggregation process of $\mathrm{RG}-\mathrm{C}_{14: 0}$, at $23^{\circ} \mathrm{C}$ and a molar ratio of FLA to host lipid of $1 / 50$ in MLV prepared from pure DPPC, DPPC/cholesterol (7/3), and POPC/cholesterol $(7 / 3)$, and in the case of RG-C $\mathrm{C}_{18: 0}$ in DPPC/cholesterol (7/3). The result is shown for the mixture of DPPC and cholesterol (7/3 molar ratio) in Fig. 4. The experimental points were well described by a theoretical curve for a second-order aggregation process [23] of the type:

$A+A \underset{k_{1}}{\stackrel{k_{2}}{\rightleftharpoons}} A_{2}$

The aggregation process has been treated as a dimerization, although there is no direct evidence to suggest that higher-order aggregation does not occur. In the case of RG-C $\mathrm{C}_{18: 0}$ (see Fig. 3E), the time-dependent appearance of two additional absorption bands (575 and $610 \mathrm{~nm}$ ) indicates, at least, that different types of aggregates are formed. However, higher-order reactions are not easily described analytically without additional assumptions and the experimental result seems to be described well by a second-order aggregation. The reaction rate constants $\left(k_{2}\right.$ and $\left.k_{1}\right)$ and the corresponding equilibrium association constant $\left(K_{\mathrm{a}}\right)$ extracted from the best fits to the

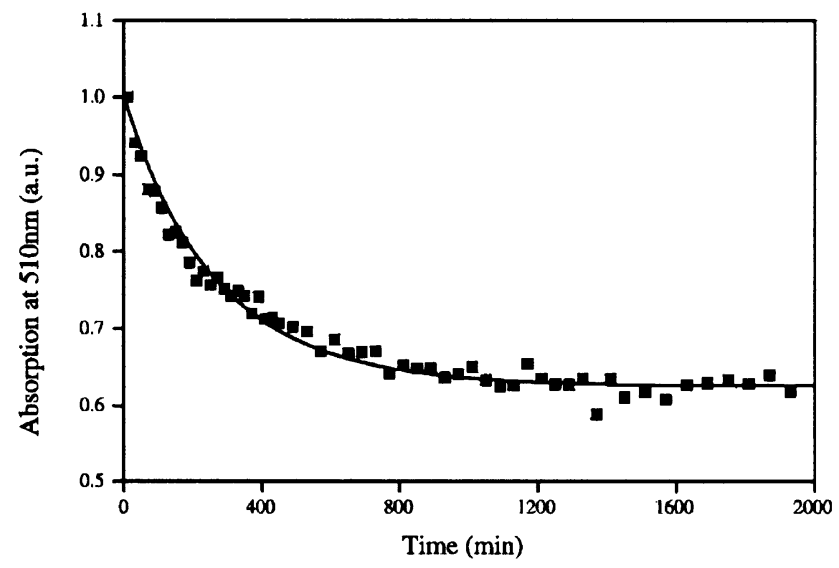

Fig. 4. Time-dependence of the aggregation of RG-tetradecylamine in DPPC/cholesterol (7/3, molar ratio) $\mathrm{MLV}$ at $23^{\circ} \mathrm{C}$ at a molar ratio of the fluorescent lipid amphiphile to the host lipid of $1 / 50$. The points are experimental data and the line is a theoretical best fit to the experimental results with $k_{2}=12.67 \mathrm{M}^{-1} \mathrm{~s}^{-1}$ and $k_{1}=2.5 \times 10^{-5} \mathrm{~s}^{-1}$ (see text for reaction scheme and definition of terms). 
Table 2

Kinetic parameters for the aggregation equilibrium of RG- $\mathrm{C}_{n: 0}$ in lipid bilayers

\begin{tabular}{llccc}
\hline FLA & Host lipid bilayer & $k_{2}\left(\mathrm{M}^{-1} \mathrm{~s}^{-1}\right)$ & $k_{1}\left(\mathrm{~s}^{-1}\right)$ & $K_{\mathrm{a}}$ \\
\hline RG-C $14: 0$ & DPPC & $3.2 \pm 1.0$ & $(3.0 \pm 0.3) \times 10^{-5}$ & $1.1 \times 10^{5}$ \\
& DPPC/cholesterol (7/3) & $13.1 \pm 1.5$ & $(1.7 \pm 0.8) \times 10^{-5}$ & $7.7 \times 10^{5}$ \\
RG-C $_{18: 0}$ & POPC/cholesterol (7/3) & $0.5 \pm 0.3$ & $(4.8 \pm 0.3) \times 10^{-5}$ & $1.0 \times 10^{4}$ \\
\hline
\end{tabular}

Results are reported as mean values \pm standard deviations of at least three separate experiments.

experimental results are summarized in Table 2. In all cases examined and listed in Table 2, equilibrium clearly favors the aggregated state of the FLA, with aggregation being most favored in the DPPC/cholesterol (7/3) bilayers and least in the POPC/cholesterol bilayers. We note that in pure POPC bilayers (liquid-disordered phase) there is no aggregation (see above). When the same probe (RG$\mathrm{C}_{14: 0}$ ) is considered, the second-order aggregation (dimerization) rate constant is fastest in the liquid-ordered phase represented by DPPC/cholesterol (7/3) and slowest in the liquid-ordered phase represented by POPC/cholesterol (7/3) bilayers at $23^{\circ} \mathrm{C}$. The first-order disaggregation rate constants are comparable in all the systems examined. It is of interest to compare the rate constants for the two liquid-ordered phases examined. Whereas the aggregation is about 25 times faster, the disaggregation process is three times slower in DPPC/cholesterol than it is in POPC/cholesterol. We may also compare the processes in pure DPPC (solid phase) and the DPPC/cholesterol (7/ 3) (liquid-ordered phase) bilayers. In the liquid-ordered phase, aggregation is about four times faster while the rate of disaggregation is comparable in the two phases. From the values of the second-order reaction rate constants, the aggregation process does not seem to be diffusion controlled in any of the systems examined.

A comparison of $\mathrm{RG}-\mathrm{C}_{14: 0}$ and $\mathrm{RG}-\mathrm{C}_{18: 0}$ in liquidordered phase DPPC/cholesterol bilayers shows that the aggregation rate constant for the FLA with the shorter alkyl chain is about twice as fast compared with the FLA with the longer chain. This may reflect a better solvation of the longer alkyl chain within the lipid bilayer. The dissociation rates are comparable.

\section{Reversibility of the $R G$ - $C_{14: 0}$ aggregation process in lipid bilayers}

When $\mathrm{RG}-\mathrm{C}_{14: 0}$-containing MLV prepared from a mixture of DPPC and cholesterol (7/3 molar ratio) that have been stored at $23^{\circ} \mathrm{C}$ for 22 days is heated to $60^{\circ} \mathrm{C}$, the two-band (apparent maxima at 515 and $576 \mathrm{~nm}$ ) spectrum of the FLA reverts to the single band (maximum at $507 \mathrm{~nm}$ ) absorption spectrum (Fig. 5). On storing at $23^{\circ} \mathrm{C}$, the two absorption bands reappear. The aggregation process can therefore be reversed by heating the DPPC/cholesterol membranes to $60^{\circ} \mathrm{C}$. The aggregation of $\mathrm{RG}-\mathrm{C}_{14: 0}$ in lipid bilayers is therefore a result of the lipid bilayer being in a liquid-ordered state

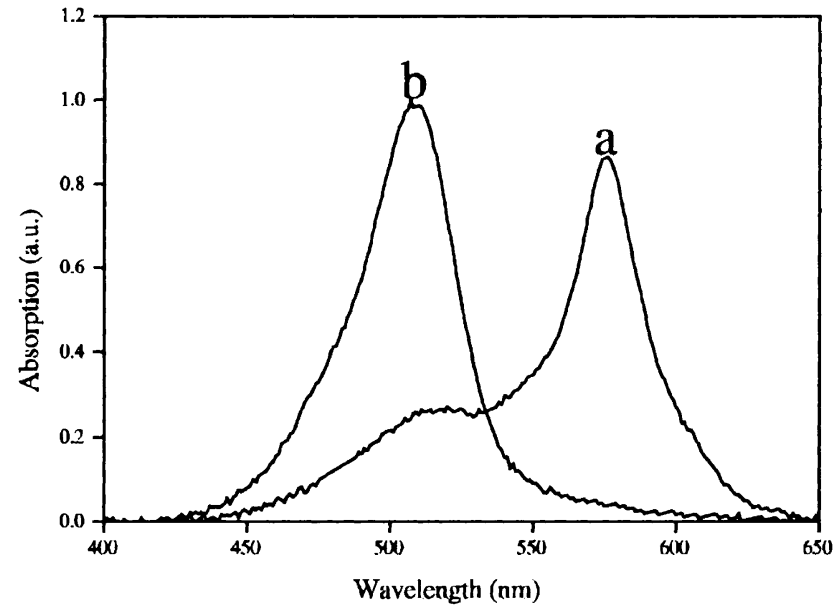

Fig. 5. Reversibility of the aggregation of RG-tetradecylamine in DPPC/cholesterol (7/3, molar ratio) bilayers at $60^{\circ} \mathrm{C}$. Traces were taken at $t=0 \mathrm{~min}$ (a) and $22 \mathrm{~h}$ (b).

and can also occur in a solid-phase bilayer but does not occur in a liquid-disordered bilayer.

Why is $R G-C_{n: 0}$ less soluble in liquid-ordered bilayer phases than $\mathrm{NBD}-\mathrm{C}_{n: 0}$ ?

As discussed earlier, the generally accepted view is that FLA solubility and partitioning in lipid bilayer membranes with phase separations depend primarily upon the nature of the apolar portion of the FLA. We present results in this work which clearly indicate a solubility difference between two FLA which have comparable apolar structures but differ in the structure and chemical properties of the polar portion which, in both cases, is also the fluorophore of the FLA. In this connection, in a recent paper [24] it was reported that while NBD-DMPE preferentially partitioned into a liquid-ordered phase (Lissamine-rhodamine B)-DMPE showed a preference for the liquid-disordered phase in membranes in which these two phases were in coexistence. In this case, too, the two FLA differed only in the nature of the fluorophore attached to the polar portion of the molecule. The reason for the difference in solubility (this work) or partitioning [24] must therefore lie in the nature of the chromophore and its interaction with the aqueous environment in which it is constrained to lie. The environment to be considered is the membrane-water interface and the aqueous phase up to a distance of about $1 \mathrm{~nm}$ above this interface. Perhaps, the 
dominant environmental characteristics in this region of the membrane are electrostatic [25] and these are, at least in part, responsible for the ordering of the aqueous phase close to the membrane surface, which results in the socalled hydration force. Since, in this work as well as that of [24], we are dealing with neutral membranes with no ionic gradients across the membrane, the surface and transmembrane potentials can be ignored and the only relevant electrostatic potential becomes the dipolar potential of the membrane. This potential is positive inside the membrane and negative towards the aqueous phase. It is, then, of interest to examine the electrostatic properties, in particular the dipole moment and its orientation relative to the membrane surface, of the FLA in question. We have calculated the theoretical dipole moments and their orientations in minimum energy conformations of the FLA used in this work using the AMI semi-empirical method included in the HyperChemVersion 5 package. For these calculations, the molecules were considered to be in a vacuum so that no solvation or other intermolecular interactions were considered. NBD- $\mathrm{C}_{16: 0}$ has a dipole moment of $9.1 \mathrm{D}$ and is negative toward the end farthest away from the membrane surface. $\mathrm{RG}-\mathrm{C}_{14: 0}$ has a dipole moment of $15.7 \mathrm{D}$ and is negative toward the membrane surface. The minimum energy conformations of the two molecules and the orientations of their dipole moments relative to the molecular structure are shown in Fig. 6. It is immediately evident that the dipolar potential of the membrane and the dipole moment of NBD- $\mathrm{C}_{16: 0}$ have the same orientation. The orientation of the dipole moment of $\mathrm{RG}-\mathrm{C}_{14: 0}$, on the other hand, is opposite that of the membrane dipolar potential. A similar argument can be made for the hydration force. This force results from a structuring and orientational ordering of the water molecules near the membrane-water interface. The orientational ordering follows the dipolar potential of the membrane so that the water dipoles may be expected to be oriented with their negative ends facing towards the bulk aqueous phase. The structured solvation layer(s) around the NBD group may be expected to have the same orientation while that around the RG group would have the opposite orientation. One might therefore expect a hydration force that drives the latter molecule out of the ordered water at the membrane interface. Since the alkyl group keeps it anchored in the membrane, the tendency to minimize the energy of the system forces the FLA into an aggregated state. Whichever way one prefers to view the system, the underlying cause for the aggregation of RG$\mathrm{C}_{14: 0}$ (and perhaps for the preferential partitioning of (Lissamine-rhodamine B)-DMPE [24]) may be attributed to the membrane dipolar potential. This interpretation also suggests that more ordered membrane phases, in particular the more ordered liquid-ordered phases such as DPPC/cholesterol (7/3), have a stronger dipolar potential than less ordered liquid-ordered phases, such as POPC/ cholesterol (7/3), or liquid-disordered phases.

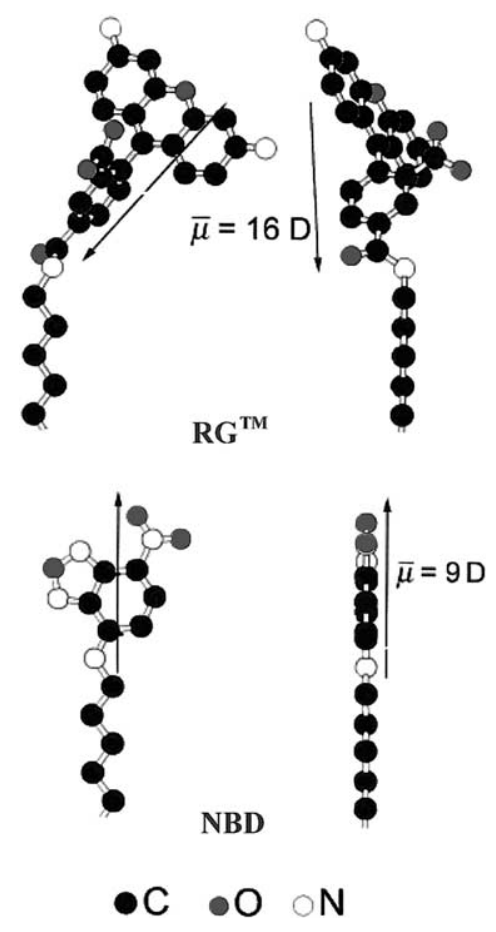

Fig. 6. Structures of RG-tetradecylamine and NBD-hexadecylamine as obtained from semi-empirical quantum mechanical calculations (AM1). Two views are presented showing the orientation of the fluorophore relative to the alkyl chain positioned parallel to the normal to the membrane plane. The orientations and magnitudes of the calculated molecular dipole moments (the arrow oriented from positive to negative) are presented with reference to the chromophores.

If this argument is valid, it may also be speculated that it could have an important role in the partitioning of lipidlinked membrane-attached proteins in the plasma membrane of cells. The orientation of helical segments of the proteins proximal to the membrane surface could present significant dipole moments whose local interaction with the dipolar surface potential of the local membrane environment will determine whether they prefer to reside in more ordered membrane domains such as "rafts."

\section{Acknowledgments}

This work was supported in part by projects funded by the Portuguese Ministry for Science and Technology (Fundação para a Ciência e a Tecnologia) through the Praxis and Sapiens programs. Luís Estronca and Magda Abreu acknowledge support in the form of stipends for initiation into scientific research (BIC) from the Fundação para a Ciência e a Tecnologia.

\section{References}

[1] M.K. Jain, H.B. White III, Long-range order in biomembranes, in: R. Paoletti, D. Kritchevsky (Eds.), Advances in Lipid Research, vol. 15, Academic Press, New York, 1977, pp. 1-60. 
[2] W.L.C. Vaz, P.F.F. Almeida, Phase topology and percolation in multi-phase lipid bilayers. Is the biological membrane a domain mosaic? Curr. Opin. Struct. Biol. 3 (1993) 482-488.

[3] K. Simons, E. Ikonen, Functional rafts in cell membranes, Nature 387 (1997) 569-572.

[4] K. Simons, E. Ikonen, How cells handle cholesterol, Science 290 (2000) 1721-1726.

[5] A. Rietveld, K. Simons, The differential miscibility of lipids as the basis for the formation of functional membrane rafts, Biochim. Biophys. Acta 1376 (1998) 467-479.

[6] D.A. Brown, E. London, Functions of lipid rafts in biological membranes, Annu. Rev. Cell Dev. Biol. 14 (1998) 111-136.

[7] D.A. Brown, E. London, Structure and function of sphingolipidand cholesterol-rich membrane rafts, J. Biol. Chem. 275 (2000) $17221-17224$.

[8] W.L.C. Vaz, Diffusion and chemical reactions in phase-separated membranes, Biophys. Chem. 50 (1994) 139-145.

[9] W.L.C. Vaz, Consequences of phase separations in membranes, in: Y. Barenholz, D. Lasic (Eds.), Handbook of Non-Medical Applications of Liposomes, Models for Biological Phenomena, vol. 2, CRC Press, Boca Raton, FL, 1996, pp. 51-60.

[10] R.Y. Tsien, The green fluorescent protein, Annu. Rev. Biochem. 67 (1998) 509-544.

[11] M.J. Saxton, K. Jacobson, Single particle tracking: application to membrane dynamics, Annu. Rev. Biophys. Biomol. Struct. 26 (1997) 373-399.

[12] J. Hwang, L.A. Gheber, L. Margolis, M. Edidin, Domains in cell plasma membranes investigated by near-field scanning optical microscopy, Biophys. J. 74 (1998) 2184-2190.

[13] C.W. Hollars, R.C. Dunn, Submicron structure in L- $\alpha$-dipalmitoylphosphatidyl-choline monolayers and bilayers probed with confocal, atomic force and near-field microscopy, Biophys. J. 75 (1998) 342-353.

[14] G.J. Schütz, G. Kada, V.P. Pastushenko, H. Schindler, Properties of lipid microdomains in a muscle cell membrane visualized by single-molecule microscopy, EMBO J. 19 (2000) 892-901.

[15] A. Pralle, P. Keller, E.L. Florin, K. Simons, J.K.H. Hörber, Sphingolipid-cholesterol rafts diffuse as small entities in the plasma membranes of mammalian cells, J. Cell Biol. 148 (2000) 997-1007.

[16] R.M.R.S. Mesquita, E. Melo, T.E. Thompson, W.L.C. Vaz, Partitioning of amphiphiles between coexisting ordered and disordered phases in two-phase lipid bilayer membranes, Biophys. J. 78 (2000) 3019-3025.

[17] A. Pokorny, P.F.F. Almeida, E.C.C. Melo, W.L.C. Vaz, Kinetics of amphiphile association with two-phase lipid bilayer vesicles, Biophys. J. 78 (2000) 267-280.

[18] A. Pokorny, P.F.F. Almeida, W.L.C. Vaz, Association of a fluorescent amphiphile with lipid bilayer vesicles in regions of solid-liquid-disordered phase coexistence, Biophys. J. 180 (2001) 1384-1394.

[19] M.S.C. Abreu, L.M.B.B. Estronca, M.J. Moreno, W.L.C. Vaz, Binding of a fluorescent lipid amphiphile to albumin and its transfer to lipid bilayer membranes, Biophys. J. (submitted).

[20] W.L.C. Vaz, E. Melo, Fluorescence spectroscopic studies on phase heterogeneity in lipid bilayer membranes, J. Fluorescence 11 (2001) 255-271.

[21] G.R. Bartlett, Phosphorus assay in column chromatography, J. Biol. Chem. 234 (1959) 466-468.

[22] R.P. Taylor, A.V. Broccoli, C.M. Grisham, Enzymatic and colorimetric determination of total serum cholesterol, J. Chem. Educ. 55 (1978) 63-64.

[23] M.J. Moreno, E. Melo, K. Razi-Naqvi, Kinetics of monomerdimer equilibria and related reactions, J. Chem. Educ. (submitted).

[24] L.M.S. Loura, A. Fedorov, M. Prieto, Fluid-fluid membrane microheterogeneity: a fluorescence resonance energy transfer study, Biophys. J. 80 (2001) 776-788.

[25] H. Brockman, Dipole potential of lipid membranes, Chem. Phys. Lipids 73 (1994) 57-79. 